\title{
Equipment for repetitive transcranial magnetic stimulation
}

\author{
Alberto M. Pernía, Candela Zorzo, Miguel J. Prieto, Juan A. Martínez, Sara G. Higarza, Marta \\ Méndez, Jorge L. Arias
}

\begin{abstract}
Repetitive transcranial magnetic stimulation (rTMS) is a non-invasive neuromodulation technique used for the treatment of a great variety of neurological disorders. The technique involves applying a magnetic field in certain areas of the cerebral cortex in order to modify neuronal excitability outside the skull. However, the exact brain mechanisms underlying rTMS effects are not completely elucidated. For that purpose, and in order to generate a pulsed magnetic field, a half-bridge converter controlled by a microcontroller has been designed to apply rTMS in small animals. Moreover, the small size of the rodent head makes it necessary to design a magnetic transducer, with the aim of focusing the magnetic field on selected brain areas using a specific and a small magnetic head. Then, our purpose was to compare the effects of five different rTMS dosages on the rat brain metabolic activity. The experimental results showed that one day of stimulation leads to an enhancement of brain metabolic activity in cortical areas, meanwhile with three days of stimulation it is possible to also modify subcortical zones, results that were not found when extending the number of rTMS applications up to seven days. In consequence, the number of pulses delivered might be an important parameter in rTMS protocols, highlighting its importance in rTMS impact.
\end{abstract}

Index Terms - Cytochrome c-oxidase, magnetic therapy, neuromodulation, repetitive transcranial magnetic stimulation.

\section{INTRODUCTION}

$\mathrm{T}$ HE transcranial magnetic stimulation (TMS) is a noninvasive technique used both to explore neurophysiological functions and to modulate cortical excitability being in the latter case, promising as a brain stimulation therapy [1]. TMS employs magnetic fields delivered by a stimulation coil located over the skull. However, those magnetic fields are converted into electric energy according to Michael Faraday electromagnetic induction principle [2]. Ultimately, this electromagnetic energy is the responsible of the physiological effects that TMS achieves [3].

The first effective transcranial electrical stimulation method was used by Merton and Morton in 1982 [4]. They showed that an electric current on the scalp was able to activate the motor cortex, producing a contraction of the contralateral muscles and that the stimulus in the visual cortex produced phosphenes. Barker and Col in 1985 designed an electromagnetic stimulator and for the first time managed to stimulate the motor cortex through the scalp [5].

Depending on the researcher/clinician purpose, the selection of the TMS modality can vary. Generally, single-pulse TMS (sTMS) and paired-pulsed TMS (ppTMS) are selected to examine brain functioning whereas repetitive TMS (rTMS) is commonly employed to the treatment of a wide variety of nervous system disorders [6]. The core reason is that rTMS effects can last beyond the stimulation period as it may promote long-term changes in neurons [7], [8].

Assuming that several psychiatric and neurological disorders show an altered neuronal connectivity, rTMS is expected to normalize the brain dysfunctional circuitry [9]. Nowadays, the main target of rTMS therapy is major depression [10], [11] and chronic pain [12]. However, other disorders such as anxiety, schizophrenia, substance use or neurodevelopmental disorders and even, neurodegenerative diseases have found benefits from its administration [13]-[15].

Nevertheless, despite the extensive variety of human studies focusing on the clinical effects using very different rTMS dosages [16], there is less research addressed to compare its impact on brain activity, although as may be expected, the dosage seems to influence rTMS modulatory effects [17]. Also, other parameters such as the intensity, magnetic waveforms, the cortical area targeted or the coil size and shape selected might be interesting [18].

Specifically, the application of rTMS requires the use of a transducer that focuses the magnetic radiation on a specific cortex area [19], [20].

This is remarkably important when conducting rTMS in animal studies, where the size of the magnetic head is a limiting factor. Thus, the geometry of the transducer will be a significant aspect in the design of the equipment, together with the intensity, frequency and repetition of the stimulus.

Thus, our aim was to compare the effects of five different rTMS protocols on the brain metabolic capacity of healthy rats. Brain metabolism was explored through quantitative 
cytochrome c oxidase (CCO) histochemistry. Cytochrome coxidase is a mitochondrial enzyme that catalyses oxygen consumption during cellular respiration, and it is involved in the oxidative phosphorylation process that generates energy [21], [22]. Interestingly, $\mathrm{CCO}$ reveals sustained changes in tissue metabolic capacity [23]. Therefore, metabolic brain activity oscillations could be assessed by the use of CCO histochemisty. Here, in each protocol, our independent variables were the number of pulses delivered -determined by different session trials and days of stimulation- and the maintenance of rTMS effect on brain metabolic activity.

\section{MAGNETIC TRANSDUCER}

There are several commercial systems designed to apply rTMS therapies. All of them are designed to radiate a large area of the human brain. For a more exhaustive analysis of the possible applications and benefits of rTMS-based therapy, it would be convenient to be able to further limit the radiated field. The use of animal models is proposed, specifically Wistar rats. These animals introduce a new problem in the study, the small size of the brain, which makes it necessary to redesign the magnetic transducer used, in order to concentrate the magnetic radiation on specific brain regions [24], [25].

Therefore, the radiation must be selective, limiting itself to the area of the brain that is going to be studied. The typical structure of the magnetic transducer is an open core winding. This geometry implies the need to use high currents in the winding to mitigate the attenuation of the magnetic field with distance. Since the geometry must be adapted to the size of the head of the rat, a new difficulty appears, the construction of a small winding meant to manage high currents. Therefore, the design of the magnetic coil or transducer has two basic limitations. On the one hand, it is desirable to reach high field values on the brain surface, but, on the other hand, it is also necessary to limit the section to be radiated.

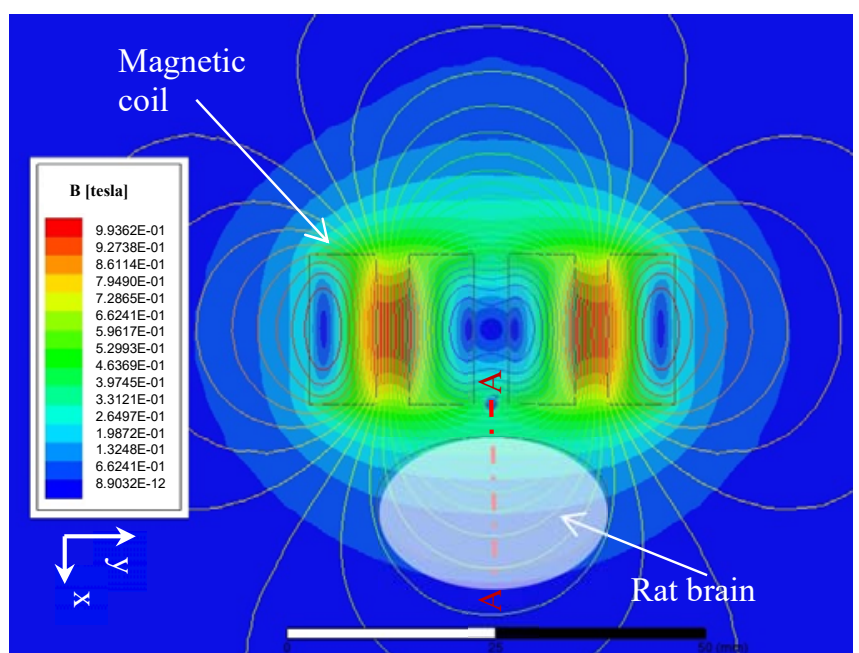

Fig. 1. Magnetic field distribution using two coils $\mathrm{I}=100 \mathrm{~A}, \mathrm{~N}=200$. Distance to the skull 5mm with ANSYS-MAXWELL.

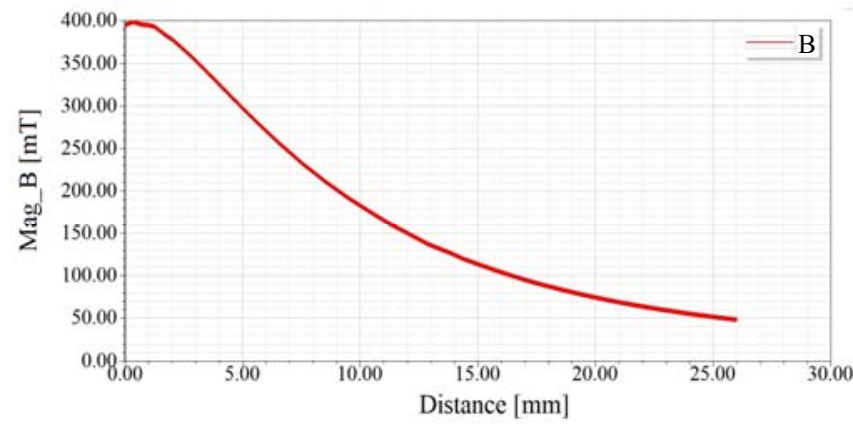

Fig. 2. Magnetic field distribution in section A-A (fig. 1)

As first alternative an air coils can be used but it makes the latter feature very difficult to achieve due to the field dispersion obtained.

In fig. 1 the distribution of the magnetic field shows the high magnetic dispersion around the brain rat when using air coils. It is very difficult to identify which are the brain regions affected by the magnetic radiation and whether there are some others which are not. The high current amplitude (100A) also results in high conduction losses. The maximum magnetic field obtained appear on the surface of the coil (400 mT) and decrease to $100 \mathrm{mT}$ in around $15 \mathrm{~mm}$.

Thus, both problems make it difficult to think of air coils as a good solution to build a small device that can focus the magnetic field and to get large field amplitudes while using low current amplitude.

The alternative proposed involves the use of a magnetic material core, aiming to conduct the magnetic flux lines to a specific region. This solution, however, involves limiting the maximum magnetic field that could be obtained to $1.5 \mathrm{~T}$ which is the typical saturation value for iron-based magnetic materials.

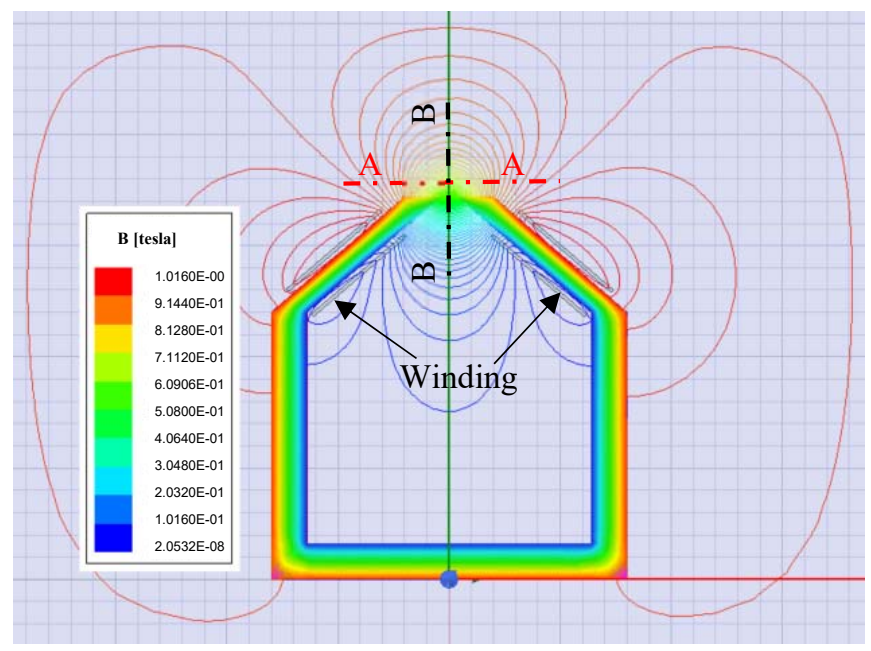

Fig. 3. Magnetic field distribution with magnetic core

Figure 3 shows a finite element analysis simulation of the geometry proposed. It represents a $U$ core of grain-oriented iron where the two columns approach each other without contacting keeping a gap distance of $5 \mathrm{~mm}$. The windings handle $3000 \mathrm{At}$ (Amps-turn). 


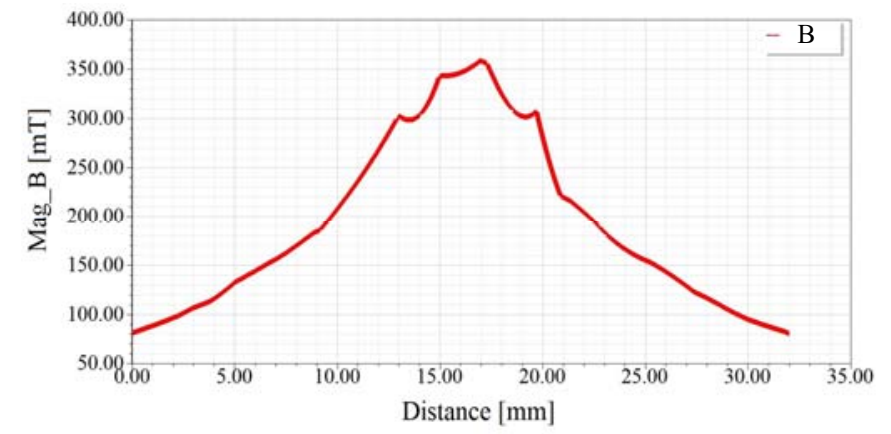

Fig. 4. Magnetic field distribution across section A-A, $2 \mathrm{~mm}$ from magnetic core

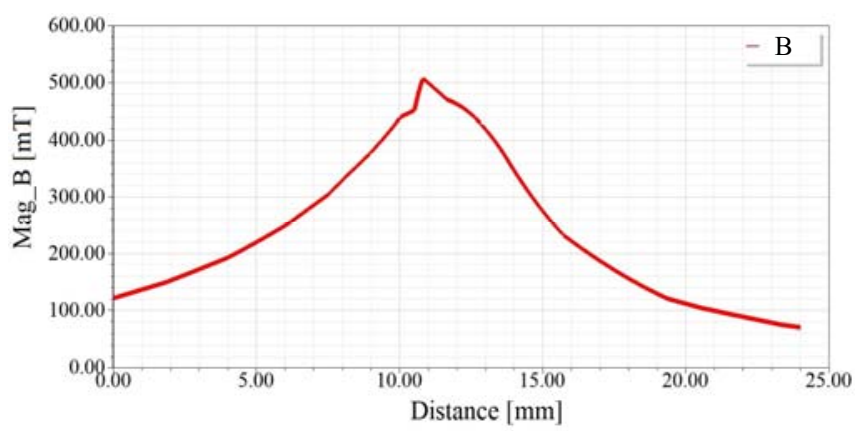

Fig. 5. Magnetic field distribution across section B-B.

The magnetic field along section A-A shows a maximum value of $0.35 \mathrm{~T}$ (Fig. 4), i.e., similar magnetic field amplitude can be obtained with this transducer while using lower currents (compared to those needed in the structure of fig. 1). The magnetic field is also expanded through the air but there is a clear direction of propagation (Fig.3, BB) defined by the flux lines in which the amplitude is maximum. Fig. 5 represents the magnetic field through the BB section and how it is attenuated with the distance to the magnetic header. Assuming around 5 $\mathrm{mm}$ to the brain cortex a magnetic field close to $200 \mathrm{mT}$ is applied. The maximum amplitude is obtained in the gap of the magnetic core.

Although the flux lines are now more concentrated around the section defined by the magnetic core, in order to be able to further limit the section that is radiated with the magnetic field, a shielding conductor $(\mathrm{Cu})$ with a thickness of $1 \mathrm{~mm}$ is used (Fig. 6). The alternating magnetic field generated will induce eddy currents in the $\mathrm{Cu}$ shielding that will partially cancel the magnetic field, thus reducing the section where it is present. As a result, a greater focus of the magnetic field is achieved.

The effectiveness of a copper foil to shield magnetic field depends mainly on absorption losses, which depends on the skin depth $(\delta)$ that for a frequency of $1.6 \mathrm{kHz}$ is $1.63 \mathrm{~mm}$. Then, the attenuation due to absorption losses (A) obtained with a $\mathrm{t}=1 \mathrm{~mm}$ copper foil can be calculated with the expression:

$$
A(d B)=20 \log e^{-t / \delta} \approx 8.7\left(\frac{t}{\delta}\right)=5.3 d B
$$

This means that the magnetic field is reduced by half using the $1 \mathrm{~mm}$ foil. Increasing the thickness of the shield will attenuate much more the whole magnetic field at the rat brain because of the higher distance. Then there is a trade-off between the distance from the magnetic material to the brain and the shielding attenuation. It is interesting to point out that the shape of the flux lines has been modified by introducing the copper shielding as we can observe comparing fig 3 and fig 6 .

The magnetic field amplitude remains almost the same along section BB (Fig. 7), but the important achievement is that the dispersion of the flux lines is drastically reduced once the $\mathrm{Cu}$ shield is reached (Fig. 8). There is a higher concentration of the magnetic field around the gap.

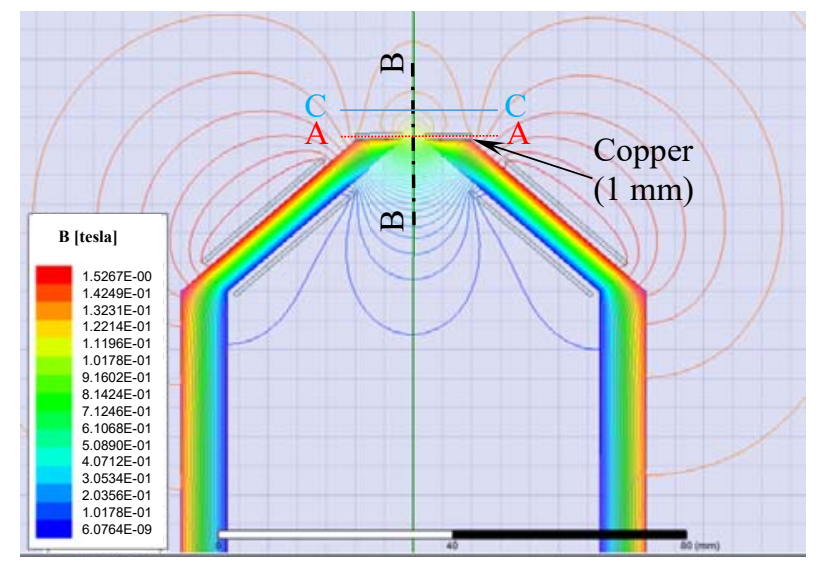

Fig. 6. Magnetic field distribution with $1 \mathrm{~mm}$ copper shield

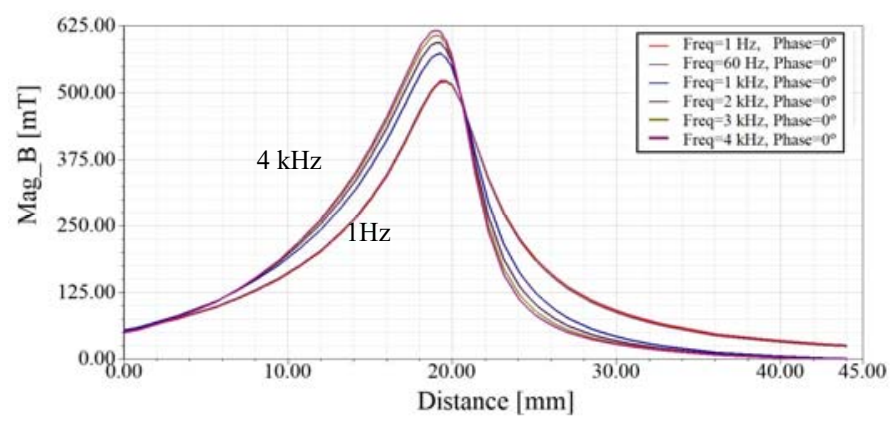

Fig. 7. Magnetic field distribution across section B-B with $1 \mathrm{~mm}$ copper shield, varying the frequency from $1 \mathrm{~Hz}$ to $4 \mathrm{kHz}$

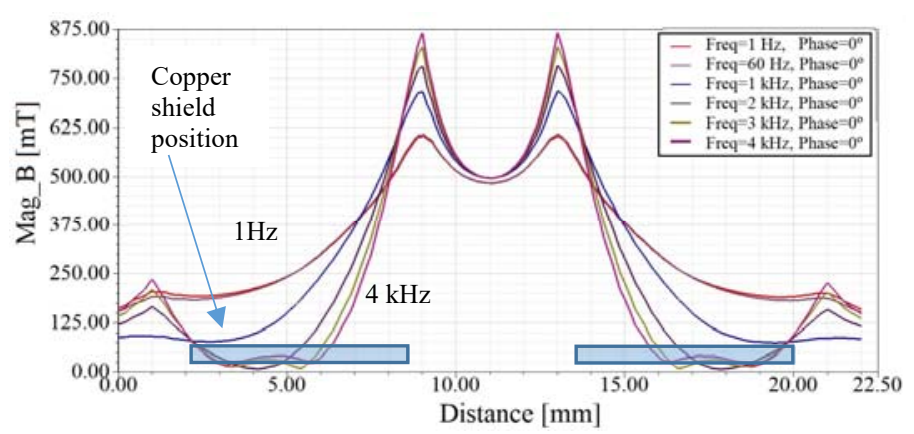

Fig. 8. Magnetic field distribution across section A-A (fig. 6) 


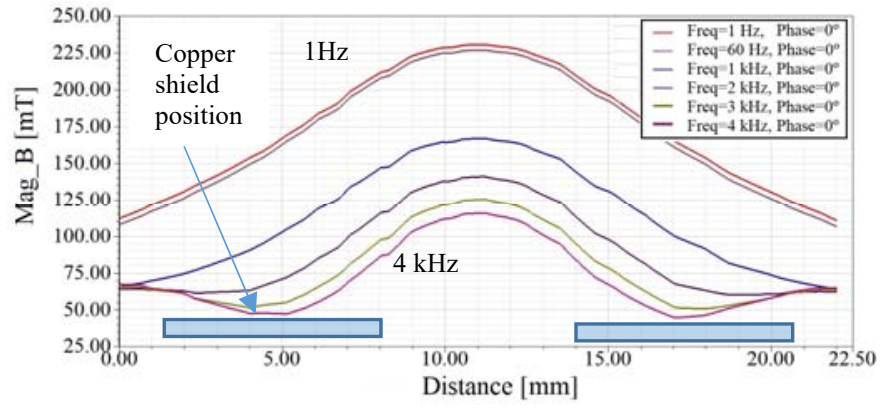

Fig. 9. Magnetic field distribution across section $\mathrm{C}-\mathrm{C}, 4 \mathrm{~mm}$ from the magnetic core (fig. 6)

This effect is clearer when the frequency in the coil is higher. The simulations shown in fig 8 and fig. 9 have been carried out with frequencies from $1 \mathrm{~Hz}$ to $4 \mathrm{kHz}$. When the distance to the core increases the effect is less evident but still patent (Fig.9).

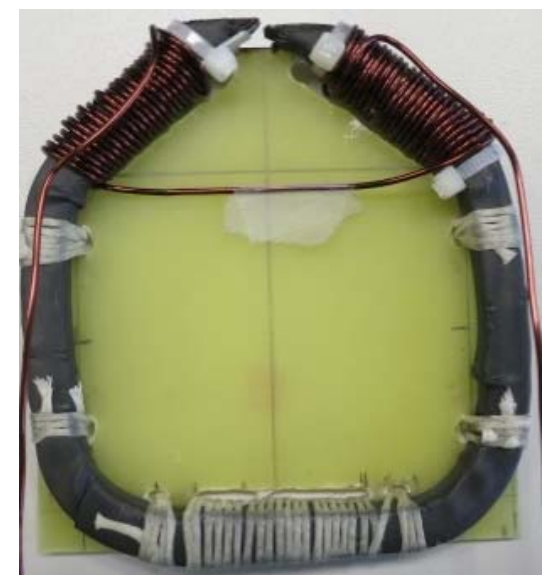

Fig. 10. Coil designed for magnetic field radiation

Thus, the inclusion of a magnetic core with a shielding like that indicated in fig. 6 provides the two initial objectives: reducing the winding current and focusing the magnetic field on a specific area. Therefore, the construction of a coil with a geometry similar to that already simulated was accepted (Fig. 10). As a first option and trying to minimize any high frequency effect and core losses a nanocrystalline material (Vitroperm $500 \mathrm{~F}$ ) with $\mathrm{B}_{\mathrm{S}}=1 \mathrm{~T}$ was selected.

Two windings of $\mathrm{N}=25$ turns were placed in each leg of the core and connected in series. The theoretical maximum current that gives rise to the saturation of the material can be calculated from the expression:

$$
i_{\max }=\frac{N \cdot A_{e} \cdot B_{S}}{L}
$$

With $\mathrm{Ae}=30 \mathrm{~mm}^{2} \mathrm{~N}=50, \mathrm{~L}=100 \mu \mathrm{H}$ and $\mathrm{B}_{\mathrm{S}}=1 \mathrm{~T}$, the current that makes the core reach the saturation is $\mathrm{i}_{\max }=15 \mathrm{~A}$. The use of ferrites is discarded since the material saturation is reached with values of $0.35 \mathrm{~T}$, making it impossible to have values close to $1 \mathrm{~T}$ in the magnetic material, which is necessary to have hundreds of $\mathrm{mT}$ in the rat brain.

\section{CONVERTER TOPOLOGY}

The activation of the coil is carried out by means of a half bridge converter controlled by a PIC microcontroller (Fig. 11), which is responsible for the management of the pulse train and the waiting times between each train of pulses [26]-[29]. The variability of these parameters will allow identifying how they affect the behaviour after the applied treatment.

By alternating the time conduction of Q1 and Q2 a positive and negative voltage is applied to the header winding, thus charging and discharging the inductor $\mathrm{L}$.

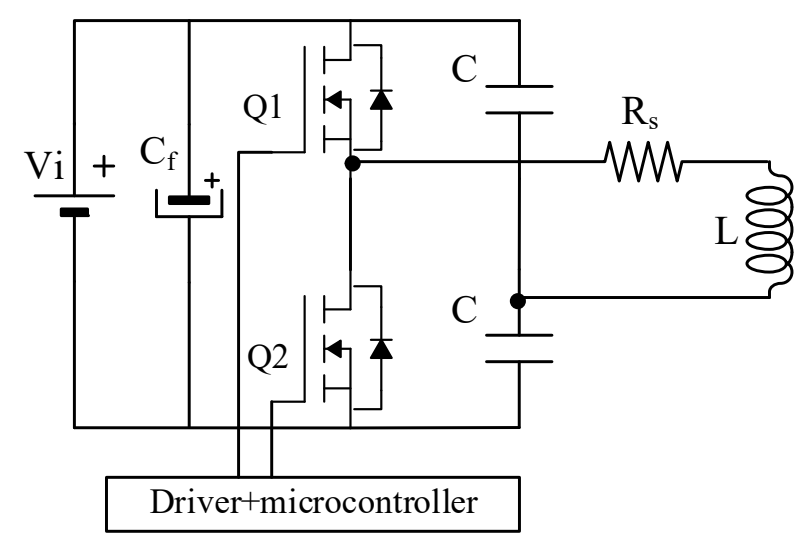

Fig. 11. Half bridge converter used in the tests.

The current through the winding (inductor L) involves the generation of an alternating magnetic field in the magnetic header [30], [31]. The amplitude of the inductor current can be controlled by adjusting the timing of switches Q1 and Q2 and the input voltage $\mathrm{Vi}$.

Not only does the microcontroller not only defines the state of transistors Q1 and Q2 to achieve the magnetic pulse, it also configures the burst pulse period.

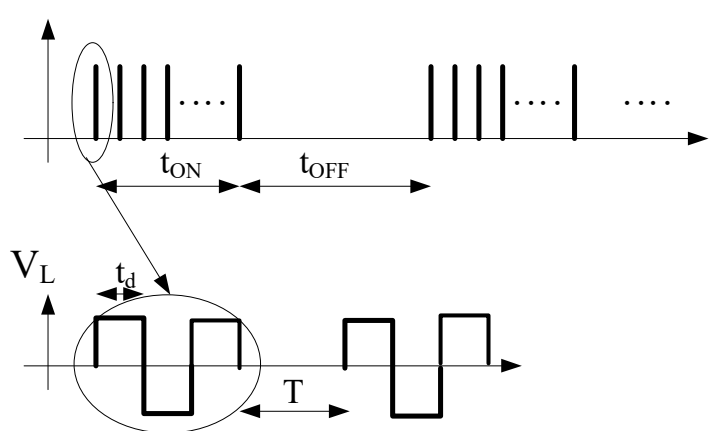

Fig. 12. Voltage pulses applied to the coil using the half-bridge topology

The prototype developed was configured to define a set of pulses during the period toN with the shape indicated in figure 12. The initial time settings are specified in the attached table I, but the study conducted focuses on the frequency of $100 \mathrm{~Hz}$, to administrate higher number of pulses in a relative few period of time, due to the limitation factor of working with animals, which need to be immobilized in order to apply rTMS without anaesthesia. 
TABLE I

PROGRAMMED TIME SETTINGS

\begin{tabular}{cll}
\hline \hline \multicolumn{1}{c}{$\mathrm{t}_{\mathrm{d}}$} & PROGRAMMED TIME SETTINGS \\
\hline $200 \mu \mathrm{s}$ & $1 / \mathrm{T}$ & toN $^{2}$ toFF $^{\mathrm{a}}$ \\
$200 \mu \mathrm{s}$ & $10 \mathrm{~Hz}$ & $5 \mathrm{~min}$ \\
$200 \mu \mathrm{s}$ & $20 \mathrm{~Hz}$ & $2.5 \mathrm{~min}$ \\
$200 \mu \mathrm{s}$ & $50 \mathrm{~Hz}$ & $1 \mathrm{~min}$ \\
\hline \hline
\end{tabular}

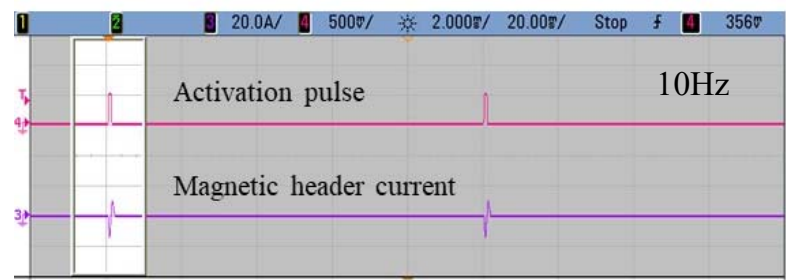

a)

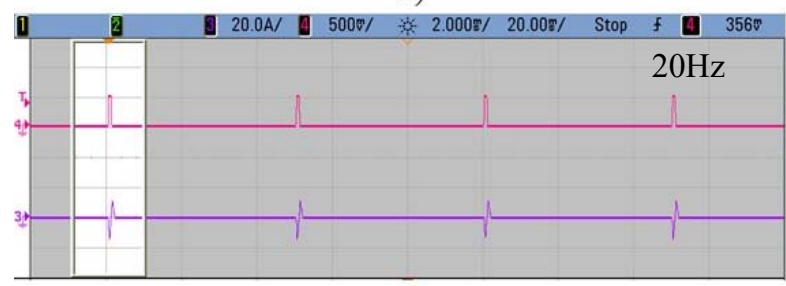

b)

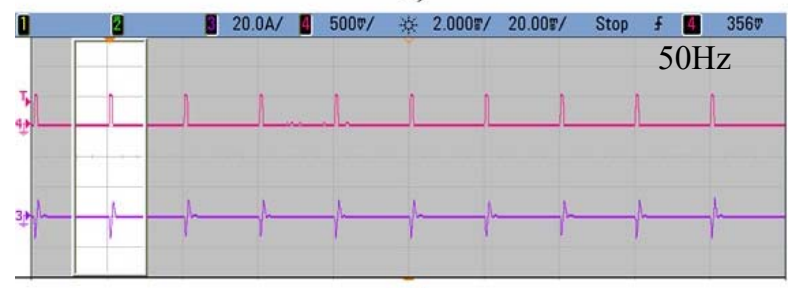

c)

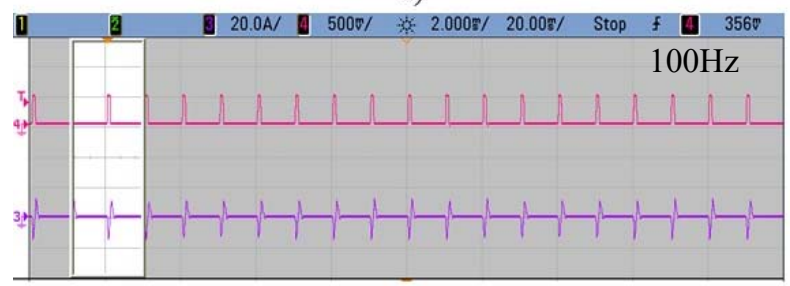

d)

Fig. 13. Activation pulses generated by the microcontroller (upper plot) and current through the magnetic header $\mathrm{i}_{\mathrm{L}}$ (lower plot) $[20 \mathrm{~A} / \mathrm{div}]$ according with table I.

Figure 13 shows the programmed activation pulse frequency according with table I and the current through the coil in the magnetic header to generate the magnetic field radiation (fig.14).

Each magnetic pulse has a frequency of $1 /\left(3 \cdot t_{d}\right)=1.6 \mathrm{kHz}$ ( fig 15 and fig. 16) and the repetition factor will be defined by $1 / \mathrm{T}$ which represents the distance between pulses. As an example, if a frequency of $100 \mathrm{~Hz}$ is selected (last row in table I), approximately 3000 pulses will be applied during $\mathrm{t}_{\mathrm{ON}}=0.5 \mathrm{~min}$.

The converter used in the tests is shown in figure 14. The need to incorporate large input capacitors $\left(\mathrm{C}_{\mathrm{f}}=1500 \mu \mathrm{F}\right)$ to transfer the pulse energy from the input capacitors to the input of the converter is emphasized.

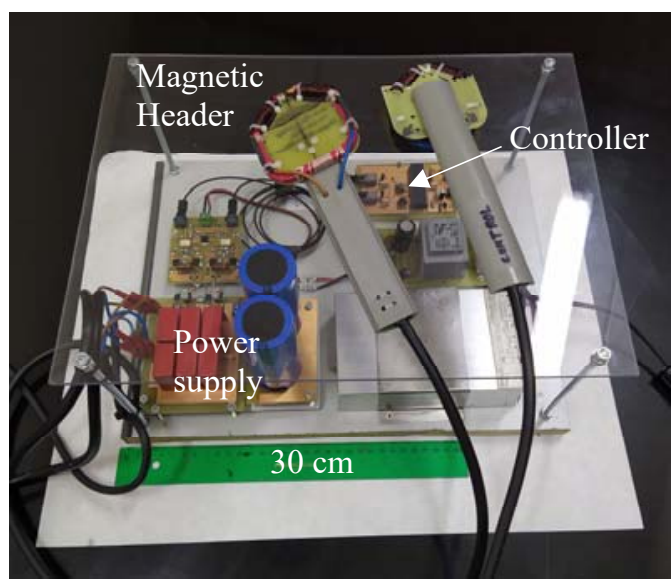

Fig. 14. Prototype developed

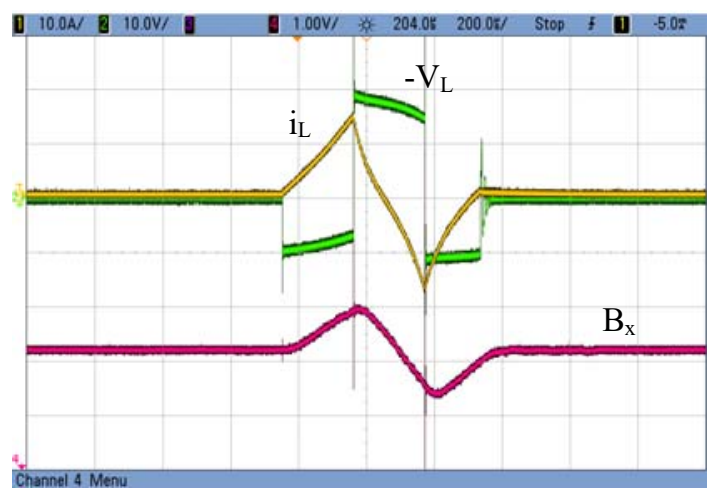

Fig. 15. Experimental results obtained with the converter shown in figure 11. The lower curve corresponds to the magnetic field at $5 \mathrm{~mm}$ from the coil (Scale $33 \mathrm{~V} / \mathrm{T})$

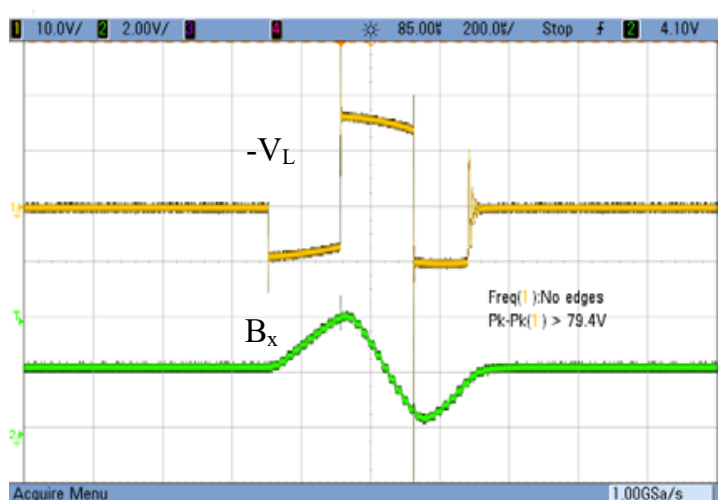

Fig. 16. Experimental results obtained with the converter shown in figure 11. The lower curve corresponds to the magnetic field at $1 \mathrm{~mm}$ of the coil (Scale $12 \mathrm{~V} / \mathrm{T}$ )

The behavior of the converter was tested using the time settings that provide a higher repetition frequency $(100 \mathrm{~Hz}$, as indicated in table I), which resulted in the curves shown in figure 15. The lower trace shows the magnetic field reached at a distance of $5 \mathrm{~mm}$ (in $\mathrm{x}$-axis direction, perpendicular to the copper shielding) from the magnetic head, $\left(\mathrm{B}_{\mathrm{x}}=30 \mathrm{mT}\right)$ with currents of 15A. For the measurement of this field, the sensor AD22151Y was used.

In figure 16 the field sensor is placed at a distance of 
approximately $1 \mathrm{~mm}$, obtaining a field amplitude $\mathrm{B}_{\mathrm{x}}=330 \mathrm{mT}$ (peak to peak).

We have selected a low intensity of magnetic induction $(0.33$ T) to see if neurophysiological changes can be found, basing on lower intensities applied in rodents could promote a reorganization of abnormal circuits [32].

\section{REPETITIVE TRANSCRANIAL MAGNETIC STIMULATION APPLICATION}

A total of 76 male young Wistar rats (200-250 g.) were used. All the animals were maintained under standard laboratory conditions (temperature: $20-22{ }^{\circ} \mathrm{C}$, relative humidity: $65-70 \%$, light-dark cycle of $12 \mathrm{~h}: 08.00-20.00 / 20.00-08.00$. The animals received ad libitum access to food and tap water. The procedures and manipulation of the animals used in this study were carried out according to the European Communities Council Directive (2010/63/UE) and the Spanish legislation on the care and use of animals for experimentation (RD 53/2013), and the study was approved by the Oviedo University committee for animal studies.
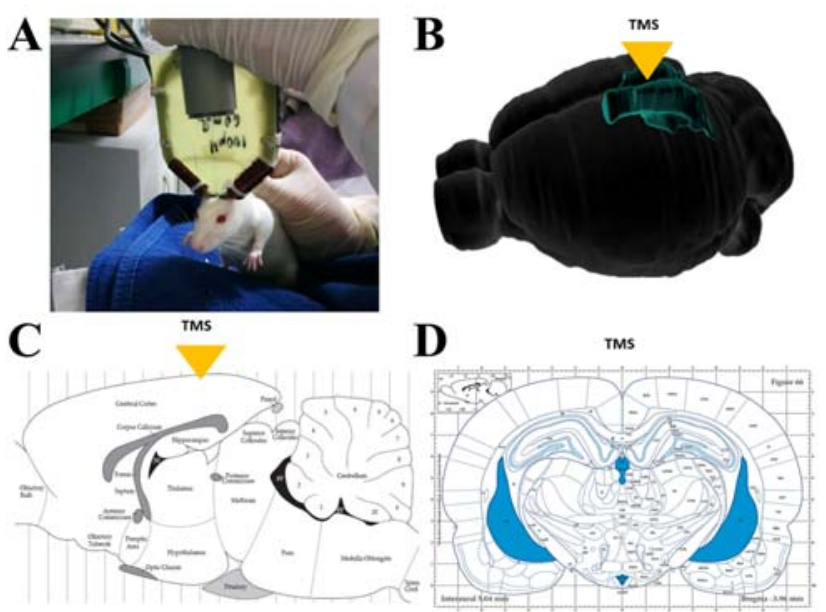

Fig. 17. (A) Application of rTMS in a Wistar rat. The magnetic field is applied in direct contact with the skull, in the retrosplenial cortex. (B) Representative image of the stimulation applied in rodent brain. Blue area indicates retrosplenial cortex (figure extracted from Blue Brain Cell Atlas). (C) Sagittal view of rat brain. (D) Coronal view of the brain according to Paxinos and Watson's atlas.

Prior to the conduct of the rTMS application, all animals were handled daily for 7 days in order to reduce the stress generated by the immobilization that the rTMS protocol requires. The rats were randomly assigned to five groups of different rTMS administration procedures and the protocol followed was the previously described by Zorzo et al., 2019 [33]. Briefly, the stimulation coil was placed midline with interaural coordinates $5.04 \mathrm{~mm}$ and bregma $-3.96 \mathrm{~mm}$ (Fig. 17) [34] at the height of the retrosplenial cortex (RSC). Before rTMS application, the skin of the animal's head was shaved. The magnetic head of the rTMS apparatus made of nanocrystalline material (Vitroperm 500F) is smaller enough to be located upper part of the previously shaved animal's head, in order to stimulate on the RSC (Fig. 17A).

In all administrations, the stimulator output delivered $0.33 \mathrm{~T}$ peak to peak with $100 \mathrm{~Hz}$ frequency. However, the procedures differ between them in the number of trials applied and in days of rTMS administration. This results in different total pulses received per group. Furthermore, groups were sacrificed 90 minutes after the last day of rTMS application except for one group which was sacrificed 48 hours after the last rTMS application (Table 2). In the procedure in which we applied 5 trials, the stimulated group received 5 minutes of trains of 100 $\mathrm{Hz}$ lasting 30 seconds each, with 30 -second intertrain intervals. In those groups in which we applied 10 trials, the stimulated group received 10 minutes of trains of $100 \mathrm{~Hz}$ lasting 30 seconds each, with 30 -second intertrain intervals. The total number of pulses delivered each minute was 3000. Each procedure was delivered in two groups: a group in which we applied transcranial magnetic stimulation (transcranial magnetic stimulated, TMS) and a control group (control, C). The $\mathrm{C}$ group was exposed to the same conditions as the experimental group, but without receiving real stimulation; for this purpose, a coil that does not emit stimulation was placed on the skull with the coordinates previously referred. The intervention was administered between 9:00 and 11:00 a.m. There were no signs of abnormal behaviour, pain or disconfort after rTMS application.

TABLE II

EXPERIMENTAL DESIGN OF REPETITIVE TRANSCRANIAL MAGNETIC STIMULATION PROCEDURE

\begin{tabular}{|c|c|c|c|c|c|c|c|}
\hline Group & $\begin{array}{l}\text { Sample } \\
\text { size }\end{array}$ & $\begin{array}{l}\text { Magnetic } \\
\text { induction }\end{array}$ & Frequency & Days & Trials & $\begin{array}{l}\text { Total } \\
\text { pulses }\end{array}$ & $\begin{array}{l}\text { Sacrifice } \\
\end{array}$ \\
\hline 5T-7D & $\begin{array}{l}\text { TMS=8; } \\
C=8\end{array}$ & $0.33 \mathrm{~T}$ & $100 \mathrm{~Hz}$ & 7 & 5 & 105000 & $90 \mathrm{~min}$ \\
\hline 10T-7D & $\begin{array}{l}\text { TMS=7; } \\
C=7\end{array}$ & $0.33 \mathrm{~T}$ & $100 \mathrm{~Hz}$ & 7 & 10 & 210000 & $90 \mathrm{~min}$ \\
\hline 10T-3D & $\begin{array}{l}\text { TMS=7; } \\
\text { C=7 }\end{array}$ & $0.33 \mathrm{~T}$ & $100 \mathrm{~Hz}$ & 3 & 10 & 90000 & $90 \mathrm{~min}$ \\
\hline 10T-1D & $\begin{array}{l}\text { TMS=8; } \\
C=8\end{array}$ & $0.33 \mathrm{~T}$ & $100 \mathrm{~Hz}$ & 1 & 10 & 30000 & $90 \mathrm{~min}$ \\
\hline $\begin{array}{l}10 T- \\
1 \mathrm{D}+2\end{array}$ & $\begin{array}{l}\text { TMS=8; } \\
C=8\end{array}$ & $0.33 \mathrm{~T}$ & $100 \mathrm{~Hz}$ & 1 & 10 & 30000 & 48 hours \\
\hline
\end{tabular}

The animals of 5T-7D, 10T-7D, 10T-3D and 10T-1D groups were euthanised 90 minutes after the end of the last stimulation session meanwhile $10 \mathrm{~T}-1 \mathrm{D}+2$ groups 48 hours after. Brains were removed and immediately frozen in isopentane (SigmaAldrich, Germany) and stored at $-40{ }^{\circ} \mathrm{C}$ to make coronal sections $30 \mu \mathrm{m}$ thick in a cryostat at $-20^{\circ} \mathrm{C}$ (Leica CM1900, Germany). The sections obtained were mounted on nongelatinized slides for $\mathrm{CCO}$ histochemistry. The regions of interest were anatomically defined according to Paxinos and Watson's atlas [34].The regions studied were included in the bregma coordinates $-3 \mathrm{~mm}$ to -5.52 for granular (RSG) and agranular retrosplenial cortex (RSA), $-2.52 \mathrm{~mm}$ to $-4.56 \mathrm{~mm}$ for the parietal cortex (PAR), $-3 \mathrm{~mm}$ for CA1, CA3 and dentate gyrus (DG) subfields of the dorsal hippocampus and $-4.56 \mathrm{~mm}$ for entorhinal (ENT) and perirhinal (PHR) cortices. Slides were processed with quantitative CCO based on González-Lima and Cada (1994) previous study [21]. The protocol was the recently explained by Banqueri et al. (2019) [35]. In brief, the sections and standards were incubated for $5 \mathrm{~min}$ in 0.1 phosphate buffer with $10 \%(\mathrm{w} / \mathrm{v})$ sucrose and $0.5(\mathrm{v} / \mathrm{v})$ glutaraldehyde, $\mathrm{pH}$ 7.6. 
After, 0.05MTris buffer, $\mathrm{pH}$ 7.6, with $275 \mathrm{mg} / \mathrm{l}$ cobalt chloride, sucrose, and $0.5(\mathrm{v} / \mathrm{v})$ dimethylsulfoxide, was applied for 10 min. Then, sections and standards were incubated in a solution with $0.0075 \%$ cytochrome-c $(\mathrm{w} / \mathrm{v}), 0.002 \%$ catalase $(\mathrm{w} / \mathrm{v}), 5 \%$ sucrose $(\mathrm{w} / \mathrm{v}), 0.25 \%$ dimethylsulfoxide $(\mathrm{v} / \mathrm{v})$, and $0.05 \%$ diaminobenzidine tetrahydrochloride (Sigma-Aldrich, Madrid, Spain), in $0.1 \mathrm{M}$ phosphate buffer at $37^{\circ} \mathrm{C}$ for $1 \mathrm{~h}$. The reaction was stopped by $4 \%(\mathrm{v} / \mathrm{v})$ formalin. Finally, the slides were dehydrated, cleared with xylene, and, cover-slipped with Entellan (Merck, Germany).

The quantification of CCO histochemical staining intensity was carried out by means of a densitometric analysis analyzing the images of the selected regions following the procedure described by Banqueri et al. (2019) [35]. For this purpose, a computer equipped with the specific analysis software MCID Core 7.0 (MCID, Interfocus Imaging Ltd., Linton, England) was employed. The average optical density of each structure was measured on the right sides of the bilateral structures using three consecutive sections with four non-overlapped readings of each animal. The values of the average optical density were transformed into units of $\mathrm{CCO}$ activity, determined by the enzymatic activity of the standards that were taken by spectrophotometry [21].

All data were analysed with the Sigma-Stat 12.5 program (Systat, Richmond, USA). A Shapiro-Wilk test was used to test the normality assumption ( $p>0.05)$. When data fit a normal distribution, a t-test for independent samples was used for the statistical comparison of CCO activity values. When the normality assumption failed, the Mann-Whitney $U$ test was applied. The results were considered statistically significant if $p<0.05$. Finally, graphic representation of the results was performed with the SigmaPlot 12.5 software program (SPSS Inc. and IBM Company, USA). Data are expressed as mean \pm SEM.

\section{RESUlts}

Figure 18 shows the mean CCO activity values measured in the 8 regions of interest of the different experimental protocols.

\section{Mean brain CCO activity 5T-7D}

The analysis of metabolic brain activity revealed no $\mathrm{CCO}$ activity differences between $\mathrm{C}$ and TMS group in PAR $\left(\mathrm{t}_{14}=\right.$ $0.178, \mathrm{p}=0.43)$, RSG $\left(\mathrm{t}_{14}=-0.166, \mathrm{p}=0.435\right)$, RSA $\left(\mathrm{t}_{14}=-0.644\right.$, $\mathrm{p}=0.265), \quad$ PHR $\quad\left(\mathrm{t}_{13}=0.837, \mathrm{p}=0.209\right)$, ENT $\quad\left(\mathrm{t}_{13}=-0.118\right.$, $\mathrm{p}=0.472), \mathrm{CA} 1\left(\mathrm{U}=27, \mathrm{n}_{1}=7, \mathrm{n}_{2}=8, \mathrm{p}=0.955\right), \mathrm{CA} 3\left(\mathrm{t}_{13}=0.643\right.$, $\mathrm{p}=0.266)$ or $\mathrm{DG}\left(\mathrm{t}_{13}=1.465, \mathrm{p}=0.0833\right)($ Fig. $18 \mathrm{~A})$.

Mean brain CCO activity 10T-7D

The analysis of metabolic brain activity revealed no CCO activity differences between $\mathrm{C}$ and TMS group in PAR $\left(\mathrm{t}_{12}=-\right.$ $0.566, \mathrm{p}=0.291), \operatorname{RSG}\left(\mathrm{t}_{12}=-0.449, \mathrm{p}=0.331\right), \mathrm{RSA}\left(\mathrm{t}_{12}=0.393\right.$, $\mathrm{p}=0.351), \quad$ PHR $\quad\left(\mathrm{t}_{11}=-0.515, \mathrm{p}=0.308\right), \quad$ ENT $\quad\left(\mathrm{t}_{11}=-0.472\right.$, $\mathrm{p}=0.323), \mathrm{CA} 1\left(\mathrm{t}_{12}=0.734, \mathrm{p}=0.238\right), \mathrm{CA} 3\left(\mathrm{t}_{12}=0.171, \mathrm{p}=0.433\right)$ or $\mathrm{DG}\left(\mathrm{t}_{12}=-0.778, \mathrm{p}=0.226\right)$ (Fig. $\left.18 \mathrm{~B}\right)$.

Mean brain CCO activity 10T-3D

The analysis of metabolic brain activity revealed higher $\mathrm{CCO}$ activity in the TMS group than in the $\mathrm{C}$ group in PAR $\left(\mathrm{t}_{12}=-\right.$ 3.919, $\mathrm{p}<0.001)$, RSG $\left(\mathrm{t}_{12}=-3.304, \mathrm{p}<0.001\right)$, RSA ( $\mathrm{U}=32$, $\left.\mathrm{n}_{1}=7, \mathrm{n}_{2}=7, \mathrm{p}=0.007\right)$ and CA1 $\left(\mathrm{t}_{12}=-3.27, \mathrm{p}<0.001\right)$ and CA3 $\left(\mathrm{t}_{12}=-1.931, \mathrm{p}=0.0387\right)$. However, no group differences were found in PHR $\left(\mathrm{t}_{12}=-0.26, \mathrm{p}=0.399\right)$, ENT $\left(\mathrm{t}_{12}=-0.256, \mathrm{p}=0.401\right)$ or $\mathrm{DG}\left(\mathrm{t}_{12}=-0.204, \mathrm{p}=0.421\right)$ (Fig. $\left.18 \mathrm{C}\right)$.
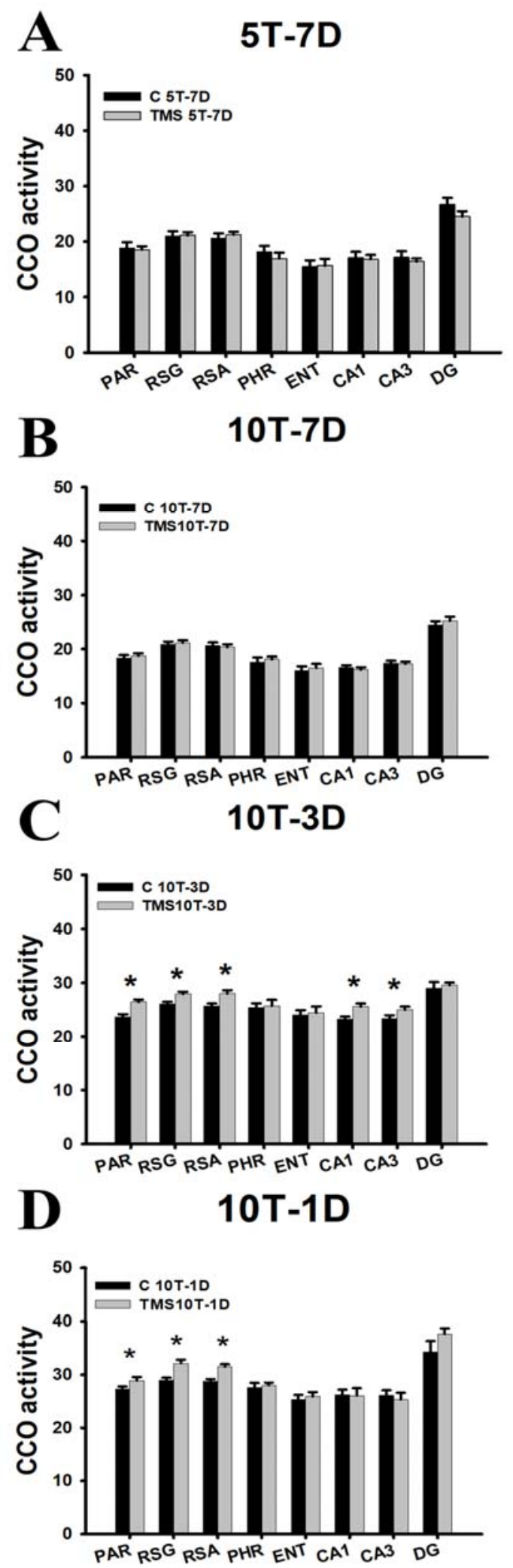


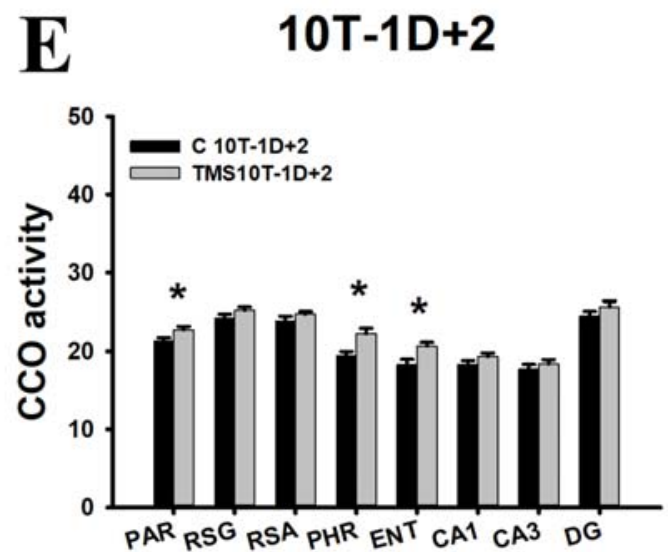

Fig 18. Cytochrome c oxidase activity values. Data represent mean $\pm \mathrm{SEM}$ (A) 5T-7D protocol. There were no significant differences in any brain region ( $>00.05$ ). (B) 10T-7D protocol. There were no significant differences in any brain region $(\mathrm{p}>0.05)$. (C). 10T-3D protocol. There were significant differences between control and stimulated animals in PAR, RSG, RSA, CA1 and CA3 $\left({ }^{*} \mathrm{p}<0.05\right)$. (D) 10T-1D protocol. There were significant differences between control and stimulated animals in PAR, RSG and RSA $\left({ }^{*} \mathrm{p}<0.05\right)$. (E) 10T$1 \mathrm{D}+2$ protocol. There were significant differences between control and stimulated animals in PAR, PHR and ENT $\left({ }^{*} \mathrm{p}<0.05\right)$. Abbreviations: parietal cortex (PAR),granular retrosplenial cortex (RSG), agranular retrosplenial cortex (RSA), perirhinal cortex (PHR), entorrinal cortex (ENT), dentate gyrus (DG).

\section{Mean brain CCO activity 10T-ID}

The analysis of metabolic brain activity revealed higher $\mathrm{CCO}$ activity in the TMS group than in the C group in PAR $\left(\mathrm{t}_{13}=-\right.$ $1,895, \mathrm{p}=0.0402)$, RSG $\left(\mathrm{t}_{13}=-4,030, \mathrm{p}<0.001\right)$, and RSA $\left(\mathrm{t}_{13}=-\right.$ $4,088, \mathrm{p}<0.001)$. However, no group differences were found in PHR $\left(\mathrm{t}_{12}=-0.403, \mathrm{p}=0.347\right)$, ENT $\left(\mathrm{U}=46, \mathrm{n}_{1}=7, \mathrm{n}_{2}=7, \mathrm{p}=0.456\right)$, CA1 $\left(\mathrm{t}_{12}=0.104, \mathrm{p}=0.459\right), \mathrm{CA} 3\left(\mathrm{t}_{12}=0.537, \mathrm{p}=0.301\right)$ or $\mathrm{DG}$ $\left(\mathrm{t}_{12}=-1.472, \mathrm{p}=0.0834\right)($ Fig. $18 \mathrm{D})$.

\section{Mean brain CCO activity 10T-ID+2}

The analysis of metabolic brain activity revealed higher $\mathrm{CCO}$ activity in the TMS group than in the C group in PAR $\left(\mathrm{t}_{14}=-\right.$ 2.375, $\mathrm{p}=0.0162)$, PHR $\left(\mathrm{t}_{12}=-3.213, \mathrm{p}<0.001\right)$ and ENT $\left(\mathrm{t}_{12}=\right.$ $2.841, \mathrm{p}<0.001)$. However, no group differences were found in RSG $\left(\mathrm{t}_{13}=-0.627, \mathrm{p}=0.0638\right)$, RSA $\left(\mathrm{t}_{13}=-0.934, \mathrm{p}=0.118\right)$, CA1 $\left(\mathrm{t}_{13}=-1.480, \mathrm{p}=0.0814\right), \mathrm{CA} 3\left(\mathrm{t}_{13}=-0.812, \mathrm{p}=0.216\right)$ or $\mathrm{DG}\left(\mathrm{t}_{12}=\right.$ $-1.285, \mathrm{p}=0.111$ ) (Fig. $18 \mathrm{E})$.

\section{DISCUSSION}

The aim of the present study was to evaluate the potential differential effects of five rTMS protocols on the brain metabolic activity of healthy rats. In particular, we examined the impact of varying the dosage, determined by different days of stimulation and session trials and also, the time interval between rTMS stimulation and the study of brain metabolic activity.

Figure 18 allows us to observe an enhancement of CCO activity in the groups that were stimulated during one and three days receiving both groups, 10 trials per day (black bars: control group; grey bars: stimulated groups). Consequently, animals of 10T-1D and $10 \mathrm{~T}-1 \mathrm{D}+2$ groups were subjected to 30000 pulses meanwhile 10T-3D group, to 90000 . However, we did not find changes in brain metabolic activity when we stimulated during 7 days, when the total number of pulses delivered were higher than 90000 , as found in $5 \mathrm{~T}-7 \mathrm{D}$ and $10 \mathrm{~T}-7 \mathrm{D}$ groups. In these cases, the total pulses administrated were 105000 for 5T-7D group and 210000 for 10T-7D group.

Three days of rTMS stimulation $(100 \mathrm{~Hz} ; 10$ trials; 90000 pulses) leads to an enhancement of $\mathrm{CCO}$ activity in the cortical area in which we stimulated, that is, RSC (both granular and agranular) but also in another cortical area located near the stimulation focus -i.e- PAR. Moreover, we have found an enhancement of CCO activity in subcortical areas such as CA1 and $\mathrm{CA} 3$ subfields of the hippocampus. These results could be explained by normal connectivity that takes place between different brain regions [36], so focal interventions such as rTMS do not have only effects on the stimulated brain area but may also affect activity in interconnected regions remote from the stimulation site [37]. Specifically, the RSC maintains connections with the hippocampal formation [38], a key structure for learning and memory [39] and also with PAR [40], a cortical region involved in a variety of cognitive processes [41].

However, with one day of stimulation $(100 \mathrm{~Hz} ; 10$ trials; 30000 pulses), we did not succeed in changing metabolic activity in subcortical structures, neither in 10T-1D group nor in 10T-1D+2. In particular, 10T-1D group showed an enhancement of CCO activity in RSC (both granular and agranular) and in PAR. These results suggest that the effects of rTMS in brain metabolism remain in cortical areas situated near the stimulation coil, maybe because only 100 minutes had elapsed (10 minutes of stimulation; 90 minutes until the euthanasia) between the beginning of the stimulation and the study of brain metabolic activity.

Interestingly, when we applied the same protocol but studied the brain metabolic activity 48 hours later (10T-1D+2 group), we saw an increment of CCO activity in PAR, ENT and PHR. In this experiment, we did not find modifications in $\mathrm{CCO}$ activity in the target of stimulation, that is, RSC. Nonetheless, as ENT and PHR are cortical regions located distant to the stimulation focus, we suggest that the increment in CCO activity observed could be due to brain functional networks. RSC maintains strong reciprocal connections with the parahippocampal area, including this region, ENT and PHR [42], [43]. Achieving the activation of parahippocampal structures far for the stimulation focus thorough rTMS administration is interesting as lateral ENT and PHR represent a functional network relevant for cognition such as object recognition and processing contextual information [43]. In human studies, it is emphasized the necessity of studying longlasting effects following rTMS applications, as the possibility of inducing perdurable effects is critical for the translation into clinical applications [44].

Finally, there are no differences in relation to controls when we increment the number of days of stimulation, and consequently, number of pulses administrated, neither in 5T-7D group (100 Hz; 5 trials; 105000 pulses) nor in 10T-7D group $(100 \mathrm{~Hz} ; 10$ trials; 210000 pulses $)$. Our results indicate that a higher number of pulses delivered (from 105000 to 210000) does not affect brain metabolism. At this point of prolonged administrations, the mitochondrial metabolic activation could present a counter-balance mechanism in order to normalize the 
metabolic pathways in the cell. It could explain why some authors do not found neither a cumulative effect [45] nor better treatment progress with higher number of pulses delivered [46]. Thus, we can suggest that a lower amount of pulses administered is recommended.

An enhancement of $\mathrm{CCO}$ activity on groups stimulated during one and three days reveals an increment on the oxidation process of cytochrome c-oxidase enzyme, which can cause an increase in oxygen consumption of cells leading to higher metabolic capacity. It could translate into a benefit at the functional level [47]. It is extremely important to note that $\mathrm{CCO}$ histochemistry allow us to visualize metabolic active regions in a restricted time frame; therefore; network interactions due to rTMS stimulation may change over time.

\section{CONCLUSION}

In summary, we can suggest that the optimal rTMS protocol of all the procedures addressed in this study is 3 days of application $(100 \mathrm{~Hz} ; 10$ sessions; 90000 pulses). With this protocol, we activate not only cortical zones but also inner structures that are essential in cognitive processes. It could be useful in order to modulate brain networks that could be altered in some experimental models [48] that aim to emulate clinical alterations in human population. In addition, we have proved that the rTMS effects of 1 day of stimulation $(100 \mathrm{~Hz} ; 10$ sessions; 30000 pulses) can endure up to 48 hours, leading to a brain metabolic activity enhancement in cortical regions distant to the stimulation focus, suggesting that rTMS effects have an impact into brain functional networks. The other goal of the present work is to design a power supply that generates a train of pulses that is configurable in terms of amplitude and frequency of repetition and that allows the previous set of experiments to be carried out. In order for this to be so, a magnetic head of small size has been designed to radiate specific areas of the head of a rat by using nanocrystalline material. Consequently, our work sums experimental evidence into the effectiveness of rTMS as a brain neuromodulation technique considering different stimulation parameters. We highlight that the parameters selected are able to influence rTMS modulatory effects in a different manner. Also, this technology still requires further study to identify which parameters influence the activation of neurons and how they do it.

\section{ACKNOWLEDGMENT}

This work was supported by PSI2017-83893-R, PSI201573111-EXP, PSI2017-90806-REDT, AINDACE Foundation and Programa "Severo Ochoa" de Ayudas Predoctorales de la Consejería de Cultura y Deporte del Principado de Asturias PA18-PF-BP17-011 to C.Z.

\section{REFERENCES}

I. Tuñez Fiñana and A. Pascual-Leone, Estimulacón magnética transcraneal y neuromodulación: Presente y futuro en neurociencias. 2014.

[2] M. Faraday, Effects on the production of electricity from magnetism. 1831.

[3] W. Klomjai, R. Katz, and A. Lackmy-Vallée, "Basic principles of transcranial magnetic stimulation (TMS) and repetitive TMS (rTMS)," Ann. Phys. Rehabil. Med., vol. 58, no. 4, pp. 208-213, 2015.

[4] P. A. Merton, H. B. Morton, D. K. Hill, and C. D. Marsden, "Scope of a Technique for Electrical Stimulation of Human Brain, Spinal Cord, and Muscle," Lancet, vol. 320, no. 8298, pp. 597-600, 1982.

A. Barker, R. Jalinous, and I. Freeston, "Non-invasive magnetic stimulation of human motor cortex.," Lancet, pp. 1106-1107, 1985.

[6] C. Zorzo, M. Banqueri, S. G. Higarza, A. M. Pernía, and J. L. Arias, "Current state of transcranial magnetic stimulation and its use in psychiatry," Actas Esp. Psiquiatr., vol. 47, no. 3, pp. 110-120, 2019. P. M. Rossini et al., "Non-invasive electrical and magnetic stimulation of the brain, spinal cord, roots and peripheral nerves: Basic principles and procedures for routine clinical and research application: An updated report from an I.F.C.N. Committee," Clin. Neurophysiol., vol. 126, no. 6, pp. 1071-1107, 2015.

[8] A. Tang, G. Thickbroom, and J. Rodger, "Repetitive Transcranial Magnetic Stimulation of the Brain: Mechanisms from Animal and Experimental Models," Neuroscientist, vol. 23, no. 1, pp. 82-94, 2015.

[9] M. Hampson, "Transcranial magnetic stimulation and connectivity mapping: tools for studying the neural bases of brain disorders," Front. Syst. Neurosci., vol. 4, no. August, pp. 1-8, 2010.

[10] H. Q. Health Quality Ontario, "Repetitive Transcranial Magnetic Stimulation for Treatment-Resistant Depression: A Systematic Review and Meta-Analysis of Randomized Controlled Trials.," Ont. Health Technol. Assess. Ser., vol. 16, no. 5, pp. 1-66, 2016.

[11] F. Rachid, "Maintenance repetitive transcranial magnetic stimulation (rTMS) for relapse prevention in with depression: A review," Psychiatry Res., no. March, pp. 0-1, 2017.

[12] B. Goudra et al., "Repetitive transcranial magnetic stimulation in chronic pain: A meta-analysis," Anesth. Essays Res., 2017.

[13] Q. Guo, C. Li, and J. Wang, "Updated Review on the Clinical Use of Repetitive Transcranial Magnetic Stimulation in Psychiatric Disorders," Neurosci. Bull., 2017.

[14] M. Q. Hameed et al., "Transcranial Magnetic and Direct Current Stimulation in Children," Curr. Neurol. Neurosci. Rep., vol. 17, no. 2, 2017

[15] A. Wagle Shukla et al., "Repetitive Transcranial Magnetic Stimulation (rTMS) Therapy in Parkinson Disease: A MetaAnalysis.," PM R, vol. 8, no. 4, pp. 356-66, 2016.

[16] J. P. Lefaucheur et al., "Evidence-based guidelines on the therapeutic use of repetitive transcranial magnetic stimulation (rTMS)," Clin. Neurophysiol., vol. 125, no. 11, pp. 2150-2206, 2014.

[17] S. Rossi et al., "Safety, ethical considerations, and application guidelines for the use of transcranial magnetic stimulation in clinical practice and research," Clinical Neurophysiology, vol. 120, no. 12. pp. 2008-2039, 2009.

[18] H. Ekhtiari et al., "Transcranial electrical and magnetic stimulation (tES and TMS) for addiction medicine: A consensus paper on the present state of the science and the road ahead," Neurosci. Biobehav. Rev., vol. 104, no. July, pp. 118-140, 2019.

[19] A. Thielscher and T. Kammer, "Electric field properties of two commercial figure-8 coils in TMS: Calculation of focality and efficiency," Clin. Neurophysiol., vol. 115, no. 7, pp. 1697-1708, 2004.

[20] L. M. Koponen, J. O. Nieminen, and R. J. Ilmoniemi, "Minimumenergy coils for transcranial magnetic stimulation: Application to focal stimulation," Brain Stimul., vol. 8, no. 1, pp. 124-134, 2015.

[21] F. Gonzalez-Lima and A. Cada, "Cytochrome oxidase activity in the auditory system of the mouse: A qualitative and quantitative histochemical study," Neuroscience, vol. 63, no. 2, pp. 559-578, 1994.

[22] M. T. T. Wong-Riley, "Cytochrome oxidase: an endogenous metabolic marker for neuronal activity," Trends Neurosci., vol. 12, no. 3, pp. 94-101, 1989.

[23] N. Arias, M. Méndez, and J. L. Arias, "Low-light-level therapy as a treatment for minimal hepatic encephalopathy: behavioural and brain assessment," Lasers Med. Sci., vol. 31, no. 8, pp. 1717-1726, 2016. Z. De Deng, S. H. Lisanby, and A. V. Peterchev, "Electric field depthfocality tradeoff in transcranial magnetic stimulation: Simulation comparison of 50 coil designs," Brain Stimul., vol. 6, no. 1, pp. 1-13, 2013.

[25] Z. De Deng, S. H. Lisanby, and A. V. Peterchev, "Coil design considerations for deep transcranial magnetic stimulation," Clin. 
Neurophysiol., vol. 125, no. 6, pp. 1202-1212, 2014.

[26] R. L. Steigerwald, "A comparison of half-bridge resonant converter topologies," IEEE Trans. Ind. Electron, vol. 3, no. 2, pp. 174-182, 2015.

[27] H. Takano, J. Takahashi, J. M. Sun, and M. Nakaoka, "Comparative study of resonant and non-resonant DC-DC converter with parasitic LC components of high-voltage transformer," Proc IEEE Ind. Appl. Soc. 33rd Annu. Meet., pp. 1580-1587, 1998

[28] J. A. Martin-Ramos, A. M. Pernía, J. Díaz, F. Nuño, and J. A. Martínez, "Power supply for a high-voltage application," IEEE Trans. Power Electron., vol. 23, no. 4, pp. 1608-1619, 2008.

[29] J. A. Martin-Ramos, A. M. Pernia, J. Diaz, F. Nuno, y J. M. Alonso, «A Circuit for the Large and Small Signal Dynamic Modeling of the PRC-LCC Resonant Topology with a Capacitor as Output Filter», en IEEE 36th Conference on Power Electronics Specialists, 2005. Aachen, Germany, 2005, pp. 635-641, doi: 10.1109/PESC.2005.1581693.

[30] A. M. Pernía, J. M. Lopera, and F. Nuño, "1D/2D transformer electric model for simulation in power converters," IEEE Power Electron. Spec. Conf. PESC'95, vol. 2, pp. 1043-1049, 1995.

[31] ]J. M. Lopera, M. Pernia, J. Diaz, J. M. Alonso, y F. Nuno, «A complete transformer electric model, including frequency and geometry effects», en PESC '92 Record. 23rd Annual IEEE Power Electronics Specialists Conference, Toledo, Spain, 1992, pp. 12471252, doi: 10.1109/PESC.1992.254732.

[32] K. Makowiecki, A. R. Harvey, R. M. Sherrard, and J. Rodger, "LowIntensity Repetitive Transcranial Magnetic Stimulation Improves Abnormal Visual Cortical Circuit Topography and Upregulates BDNF in Mice," J. Neurosci., 2014.

[33] C. Zorzo, S. G. Higarza, M. Méndez, J. A. Martínez, A. M. Pernía, and J. L. Arias, "High frequency repetitive transcranial magnetic stimulation improves neuronal activity without affecting astrocytes and microglia density," Brain Res. Bull., vol. 150, pp. 13-20, 2019.

[34] G. Paxinos and C. Watson, The rat brain in stereotaxic coordinates. Elsevier Academic Press, 2005.

[35] M. Banqueri, J. A. Martínez, M. J. Prieto, S. Cid-Duarte, M. Méndez, and J. L. Arias, "Photobiomodulation rescues cognitive flexibility inearly stressed subjects," Brain Res., vol. 1720, no. March, p. 146300,2019

[36] L. Cárdenas-Morales et al., "Network connectivity and individual responses to brain stimulation in the human motor system," Cereb. Cortex, vol. 24, no. 7, pp. 1697-1707, 2014.

[37] Á. Pascual-Leone and J. M. Tormos-Muñoz, "Estimulación magnética transcraneal: Fundamentos y potencial de la modulación de redes neurales específicas," in Rev Neurolog, 2008, vol. 46, no. SUPPL. 1.

[38] A. S. Alexander, L. M. Rangel, D. Tingley, and D. A. Nitz, "Neurophysiological signatures of temporal coordination between retrosplenial cortex and the hippocampal formation.," Behav. Neurosci., vol. 123, no. 5, pp. 453-468, 2018.

[39] J. P. Aggleton, "Multiple anatomical systems embedded within the primate medial temporal lobe: Implications for hippocampal function," Neurosci. Biobehav. Rev., vol. 36, no. 7, pp. 1579-1596, 2012.

[40] T. Oess, J. L. Krichmar, and F. Rohrbein, "A computational model for spatial navigation based on reference frames in the hippocampus, retrosplenial cortex, and posterior parietal cortex," Front. Neurorobot., vol. 11, no. 4, 2017.

[41] J. C. Myskiw and I. Izquierdo, "Posterior parietal cortex and longterm memory: some data from laboratory animals," Front. Integr. Neurosci., vol. 6, pp. 1-7, 2012.

[42] T. P. Todd and D. J. Bucci, "Retrosplenial Cortex and Long-Term Memory: Molecules to Behavior," Neural Plast., vol. 414173, 2015.

[43] J. Sugar and M. P. Witter, "Postnatal development of retrosplenial projections to the parahippocampal region of the rat," Elife, vol. 5, pp. 1-29, 2016.

[44] M. Dileone, L. Mordillo-Mateos, A. Oliviero, and G. Foffani, "Longlasting effects of transcranial static magnetic field stimulation on motor cortex excitability," Brain Stimul., vol. 11, no. 4, pp. 676-688, 2018.

[45] L. Brusa et al., "Low frequency rTMS of the SMA transiently ameliorates peak-dose LID in Parkinson's disease," Clin. Neurophysiol., vol. 117, no. 9, pp. 1917-1921, 2006.

[46] S. Teng et al., "High-frequency repetitive transcranial magnetic stimulation over the left DLPFC for major depression: Session- dependent efficacy: A meta-analysis," Eur. Psychiatry, vol. 41, pp. 75-84, 2017.

[47] J. C. Rojas, A. K. Bruchey, and F. Gonzalez-Lima, "Low-level light therapy improves cortical metabolic capacity and memory retention," J. Alzheimer's Dis., vol. 32, no. 3, pp. 741-752, 2012.

[48] G. Zanirati et al., "Depression comorbidity in epileptic rats is related to brain glucose hypometabolism and hypersynchronicity in the metabolic network architecture," Epilepsia, vol. 59, no. 5, pp. 923934, 2018. 